\title{
Theory of Anodic Stripping Voltammetry at Wall-Jet Electrodes. Simulation of Spatially Differential Stripping and Redeposition Phenomena
}

\author{
Jon C. Ball and Richard G. Compton* \\ Physical and Theoretical Chemistry Laboratory, Oxford University, South Parks Road, \\ Oxford OX1 3QZ, United Kingdom \\ Christopher M. A. Brett \\ Departamento de Quimica, Universidade de Coimbra, 3049 Coimbra, Portugal \\ Received: June 25, 1997; In Final Form: October 20, $1997^{\otimes}$
}

\begin{abstract}
Numerical simulations based on the time dependent backward implicit method are used to develop the theory of anodic stripping voltammetry carried out under hydrodynamic conditions using mercury thin film wall-jet electrodes. The peak shape is shown to be highly sensitive both to electrolyte flow rate and to the potential sweep rate. The simulations permit visualization of concentrations both in solution and in the film throughout the voltammetric potential sweep. They reveal the film to be stripped in a spatially nonuniform fashion with the flow inducing the center of the electrode to be depleted before, and at less positive potentials than, the radial extremities of the electrode. Moreover for electrochemically reversible systems with flow and sweep rate parameters similar to those employed in analytical practice it is seen that material oxidized from the electrode center can become redeposited at radial distances closer to the electrode edge where the diffusion layer is thicker, before being re-oxidized later on in the potential sweep at more positive potentials.
\end{abstract}

\section{Introduction}

Anodic stripping voltammetry (ASV) is, by virtue of its sensitivity and selectivity, an attractive approach to the analytical determination of a wide range of trace metals. ${ }^{1,2}$ The first stage in the procedure involves the accumulation and hence preconcentration of the target metals by electroreduction of the corresponding ions from aqueous solution usually at mercury electrodes so as to generate an amalgam. The second stage, the stripping step, is informative and is most simply conducted using a potential sweep so that the material deposited in the accumulation step undergoes electrooxidation. The magnitude of the associated Faradaic current may then be related to the amount of material deposited, permitting quantitative determination of the target. While both the deposition and stripping steps are readily undertaken in quiescent solution, there can be advantages in terms of sensitivity and reproducibility to adopt conditions in which the transport to the electrode is dominated by convection rather than diffusion. ${ }^{2-11}$ Moreover for many applications it is important that the electrode is located within a flow system to permit continuous monitoring and to enhance the speed of the analysis. ASV has been conducted successfully at mercury film electrodes in both the wall-jet ${ }^{5-9}$ and channel electrode ${ }^{10,11}$ geometries. Accordingly, the question arises as to whether the introduction of convection as a dominating contribution to the transport leads to any significant changes in the associated voltammetric theory.

The theory of ASV at hydrodynamic electrodes has hitherto been little developed. The work of Roe and Toni, ${ }^{12}$ of Brett and Oliveira Brett, ${ }^{13}$ and of Schiewe ${ }^{14}$ employed the Nernst diffusion layer model to establish an approximate expression for the time $(t)$ dependence of the stripping current, $I$, due to

${ }^{\otimes}$ Abstract published in Advance ACS Abstracts, December 1, 1997. the oxidation induced by a positive potential sweep from an

$$
\operatorname{Red}_{\text {film }}-n \mathrm{e}^{-} \rightleftharpoons \mathrm{Ox}_{(\mathrm{aq})}
$$

initial potential of $E_{\mathrm{i}}$ at a scan rate, $v / \mathrm{V} \mathrm{s}^{-1}$. In particular

$$
I=Z(\sigma t) n F A \sigma l[\mathrm{Red}]_{\mathrm{film}}
$$

where $\sigma=(n F / R T) v, l$ is the mercury film thickness, $[\text { Red }]_{\text {film }}$ is the concentration of metal in the mercury film, and $A$ is the electrode area. The dimensionless function $Z(\sigma t)$ has a maximum value of 0.3679 at the peak potential ${ }^{12}$ and has been tabulated. ${ }^{13}$

The diffusion layer theory predicts that for convective conditions, in comparison with stationary solution, the ASV peak becomes sharper, asymmetric in shape and shifted from the formal potential, $E^{\circ}$, by an amount

$$
E_{\text {peak }}-E^{\circ \prime}=(R T / n F) \log \left\{\delta l \sigma / D_{\text {ox }}\right\}
$$

whereas under zero convection conditions ${ }^{15}$

$$
I_{\text {peak }}=0.2975 n F A \sigma l[\operatorname{Red}]_{\text {film }}
$$

In these equations $D_{\text {ox }}$ is the diffusion coefficient of the metal ion in aqueous solution and $\delta$ is the diffusion layer thickness assumed to be uniform across the electrode area.

The derivation of eqs $1-5$ makes three key assumptions. ${ }^{12,13}$ The first is that diffusion within the mercury film can be neglected. Given the high magnitude of the reported values ${ }^{16}$ of the diffusion coefficient for various metals in mercury combined with the thin layers of mercury (generally $<1 \mu \mathrm{m}$ ) typically employed experimentally, ${ }^{1,2}$ this assumption is thought to be a very good approximation. ${ }^{12}$ Second the derivations adopt 
the diffusion layer theory, ${ }^{12,13}$ which requires that the flux of Ox leaving the electrode surface is matched exactly by transport across a linear concentration gradient instantaneously established across the full diffusion layer thickness. In a previous paper ${ }^{17}$ we have brought simulation methods to bear on the hydrodynamic ASV problem for the first time and have shown that for sufficiently high potential scan rates or thick diffusion layers this approximation is invalid and that the conditions under which the breakdown occurs are not uncommon in experimental practice. The third assumption is that the electrode is uniformly accessible such as is found for rotating disk or microhemisphere electrodes. However, as noted above, much ASV is usefully conducted under flowing conditions where the current density will, as a result of the influx of fresh electroactive material, be greater at the upstream edge of the electrode than at the downstream edge. In the context of the specific case of channel electrodes we have simulated the ASV behavior ${ }^{18}$ and shown that the effect of the flow is, in the case of electrochemically reversible Red/Ox systems, to induce spatially differential stripping of the film so that Red is oxidized out of the upstream part of the film at lower potentials than are required in the downstream part of the film. However since the diffusion layer in a channel electrode varies only as the cube root of the axial distance along the channel, the resulting effect as revealed in the voltammetry is rather weak.

The purpose of the present paper is to explore the effect of flow on ASV in the context of an electrode with grossly nonuniform accessibility, namely the wall-jet electrode. This is a well-characterized hydrodynamic electrode in which the flow is due to a (submerged) fluid jet which strikes a planar disk electrode at right angles and spreads out radially over that surface, the fluid outside the jet being at rest. ${ }^{19}$ The mass transport experienced by the electrode is dependent on its size relative to the impinging jet. The term "wall-jet"19-24 as opposed to "wall-tube" 25 corresponds to the case where the electrode is substantially larger than the jet. Such electrodes have diffusion layer thicknesses which increase as the (5/4)th power of the radius and for which effects of convection may be expected to be correspondingly amplified.

\section{Theory}

We consider the ASV stripping peak arising from the electrode reaction

$$
\operatorname{Red}_{\text {film }}-n \mathrm{e}^{-} \rightleftharpoons \mathrm{Ox}_{(\mathrm{aq})}
$$

in which the electrode potential is swept in a positive direction starting from an initial $(t=0)$ potential $E_{\mathrm{i}}$ :

$$
E=E_{\mathrm{i}}+v t \quad(t>0)
$$

If the electrode process is electrochemically reversible, then the Nernst equation applies to the concentrations of Ox and Red at the electrode surface.

$$
[\mathrm{Red}]_{\mathrm{film}} /[\mathrm{Ox}]_{(\mathrm{aq})}=\exp \left\{(-n F / R T)\left(E-E^{\circ \prime}\right)\right\}
$$

The initial concentrations $[\mathrm{Red}]_{\mathrm{film}, t=0}$ and $[\mathrm{Ox}]_{(\mathrm{aq}), t=0}$ will also obey eq 8 .

$$
[\operatorname{Red}]_{\text {film }, t=0} /[\mathrm{Ox}]_{(\mathrm{aq}), t=0}=\exp \left\{(-n F / R T)\left(E_{\mathrm{i}}-E^{\mathrm{o \prime}}\right)\right\}
$$

But usually $E_{\mathrm{i}}$ is chosen to be adequately negative of $E^{\circ \prime}$ that $[\mathrm{Ox}]_{(\mathrm{aq}), t=0} \approx 0$. The concentration of $\mathrm{Ox}$ in bulk solution is assumed to be maintained at the low value $[\mathrm{Ox}]_{(\mathrm{aq}), t=0}$ throughout

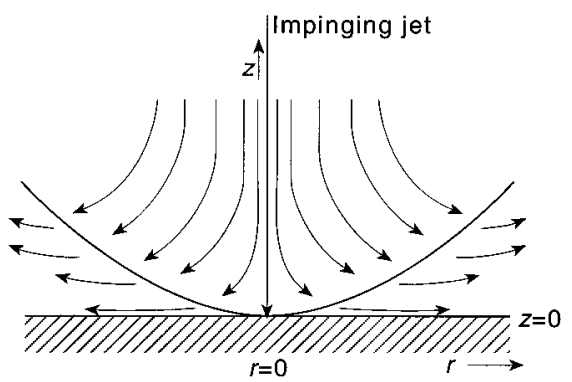

Figure 1. Flow pattern at a wall-jet electrode.

the potential sweep; this ensures that zero current flows at the starting potential in the simulations reported below.

If the mercury film is sufficiently thin that transport throughout its depth, $l$, is so fast that $[\text { Red }]_{\text {film }}$ is always uniform, then

$$
l \frac{\partial[\mathrm{Red}]}{\partial t}=D_{\mathrm{Ox}}\left(\frac{\partial[\mathrm{Ox}]}{\partial z}\right)_{z=0}
$$

where $z$ is the Cartesian coordinate normal to the electrode surface defined in Figure 1.

The transport of $\mathrm{Ox}$ in solution will be controlled by combined diffusion and convection in the wall-jet flow cell: ${ }^{26}$

$$
\frac{\partial[\mathrm{Ox}]}{\partial t}=D_{\mathrm{Ox}} \frac{\partial^{2}[\mathrm{Ox}]}{\partial z^{2}}-v_{z} \frac{\partial[\mathrm{Ox}]}{\partial z}-v_{r} \frac{\partial[\mathrm{Ox}]}{\partial r}
$$

where $v_{r}$ is the radial solution velocity ( $r$ direction) and $v_{z}$ is the velocity in the direction normal to the electrode surface $(z$ direction). Expressions for $v_{r}$ and $v_{z}$ for wall-jet flow have been derived. ${ }^{27}$ Note that in writing eq 8 radial diffusion has been neglected; the basis of this approximation is developed in ref 27. We also assume the presence of sufficient supporting electrolyte that migration effects are negligible.

Equations 7 and 8, together with the boundary conditions, eqs $4-6$ are readily solved using the time dependent backward implicit method ${ }^{28}$ essentially in the form established for linear sweep voltammetric problems in the wall-jet geometry. ${ }^{29}$ However this simulation cannot be carried out in normal Cartesian space due to the gross nonuniformity of the electrode. The $z$-coordinate must be transformed accordingly; otherwise the number of points required to simulate the full $(r, z)$ space is prohibitively large. The chosen transformation is such that the transformed coordinate, $\eta$, expands across the diffusion layer such that

$$
\begin{aligned}
& \eta=A z / r^{5 / 4} \text { where } \\
& \qquad A=\left\{135 M / 32 v^{3}\right\}^{1 / 4} \text { and } M=k_{\mathrm{e}} 4 V_{\mathrm{f}} 3 / 2 \pi^{3} a^{2}
\end{aligned}
$$

where $v$ is the kinematic viscosity, $a$ is the nozzle diameter, $V_{\mathrm{f}}$ is the volume flow rate, and $k_{\mathrm{e}}$ is an experimental parameter approximating to $0.9 .^{22}$

The maximum value of $\eta$ simulated was chosen to be 0.4 corresponding to the full diffusion layer. ${ }^{26}$ Moreover since radial diffusion effects are neglected, simulations need only be constrained to the range

$$
0<r<r_{\mathrm{e}}
$$

where $r_{\mathrm{e}}$ is the disk radius. The $(r, \eta)$ space of interest is filled by a simulation mesh of spacings,

$$
\Delta r=r_{\mathrm{e}} / K \text { and } \Delta \eta=0.4 / J
$$


The notation $[\mathrm{Ox}]_{j, k}$ is adopted to signify the concentration of $\mathrm{Ox}$ at the point $r=k \Delta r$ and $\eta=j \Delta \eta$; likewise [Red $]_{0, k}$ gives the Red concentration in the film electrode at the radial point $r$ $=k \Delta r$. In both cases a superscript $t$ indicates a time $t \Delta t$ after the start of the anodic potential sweep, where $\Delta t$ is a chosen time increment. The resulting finite difference equations obtained after transformation into finite difference form are

$$
\begin{aligned}
& \left(-\lambda_{k}^{\eta}-\lambda_{j, k}^{v_{z}}\right)[\mathrm{Ox}]_{j-1, k}^{t}+\left(\lambda_{j, k}^{v_{r}}+2 \lambda_{k}^{\eta}+1\right)[\mathrm{Ox}]_{j, k}^{t}+ \\
& \left(\lambda_{j, k}^{v_{z}}-\lambda_{k}^{\eta}\right)[\mathrm{Ox}]_{j+1, k}^{t}=[\mathrm{Ox}]_{j, k}^{t-1}+\lambda_{j, k}^{v_{r}}[\mathrm{Ox}]_{j, k-1}^{t}
\end{aligned}
$$

with the surface boundary condition transforming to

$$
\left(\exp \left(-\frac{n F}{R T} E-E^{\circ \prime}\right)+\kappa_{k}\right)[\mathrm{Ox}]_{0, \mathrm{k}}^{t}-\kappa_{k}[\mathrm{Ox}]_{1, k}^{t}=[\mathrm{Red}]_{0, k}^{t-1}
$$

where

$$
\kappa_{k}=\frac{D_{\mathrm{Ox}} \Delta t}{l \Delta \eta} \frac{A}{r^{5 / 4}} ; \quad \lambda_{k}^{\eta}=\frac{D_{\mathrm{Ox}} \Delta t}{(\Delta \eta)^{2}}\left(\frac{A}{r^{5 / 4}}\right)^{2}
$$

and

$$
\lambda_{j, k}^{v_{r}}=\frac{v_{r} \Delta t}{\Delta r} ; \quad \lambda_{j, k}^{v z}=\frac{\Delta t}{2 \Delta \eta}\left(v_{z} \frac{A}{r^{5 / 4}}-v_{r} \frac{5 \eta}{4 r}\right)
$$

The other boundary conditions for the ASV problem are 2 -fold. First, at $j=J$ bulk solution is attained such that

$$
[\mathrm{Ox}]_{j, k}^{t}=[\mathrm{Ox}]_{\mathrm{bulk}}=[\mathrm{Ox}]_{(\mathrm{aq}), t=0}
$$

Second, at $k=0$, corresponding to the center of the electrode, fresh solution is flowing into the cell such that

$$
[\mathrm{Ox}]_{j, 0}^{t}=[\mathrm{Ox}]_{\mathrm{bulk}}
$$

The equations can be combined into a single matrix equation, which is solved as described elsewhere. ${ }^{18,26}$

All programs were written in FORTRAN 77 and executed on a Silicon graphics workstation. The parameters $J, K$, and $\Delta t$, which control the spatial and temporal nodal spacing of the simulation points, were adjusted for convergence in the current to within $1 \%$; typical values were $J=1000, K=100$ and $\Delta t$ $=10^{-3} \mathrm{~s}$.

\section{Results and Discussions}

Simulations were conducted with the following parameters unless otherwise stated: $E^{\circ \prime}=-0.5 \mathrm{~V}, E_{\mathrm{i}}=-1.0 \mathrm{~V}, D_{\mathrm{Ox}}=$ $10^{-5} \mathrm{~cm}^{2} \mathrm{~s}^{-1}, l=0.1 \mu \mathrm{m}, r_{\mathrm{e}}=0.2 \mathrm{~cm}, V_{\mathrm{f}}=10^{-2} \mathrm{~cm}^{3} \mathrm{~s}^{-1}, a$ $=0.0345 \mathrm{~cm}, v=0.0089 \mathrm{~cm}^{2} \mathrm{~s}^{-1}$ (corresponding to water at $\left.25^{\circ} \mathrm{C}\right),{ }^{29} k_{\mathrm{e}}=0.9,{ }^{5,21,22}[\mathrm{Red}]_{\mathrm{film}, t=0}=1 \mathrm{M}$, and $n=2$. These values approximate to those used in analytical practice.

Stripping voltammograms were simulated for a range of potential scan rates. Qualitatively similar effects were observed as noted for channel electrodes; ${ }^{18}$ at fast scan rates the peaks become less asymmetric and more closely resemble those that would be found in stationary solution. ${ }^{11}$ Comparisons of the simulations with reported experimental data $^{5-9}$ support the veracity of the calculations and encourages their use for interpreting analytical data. However the main purpose of this paper is to explore the effect of the gross nonuniform accessibility of the wall-jet electrode especially in the context of spatially differential stripping. It is to this aspect we turn next.

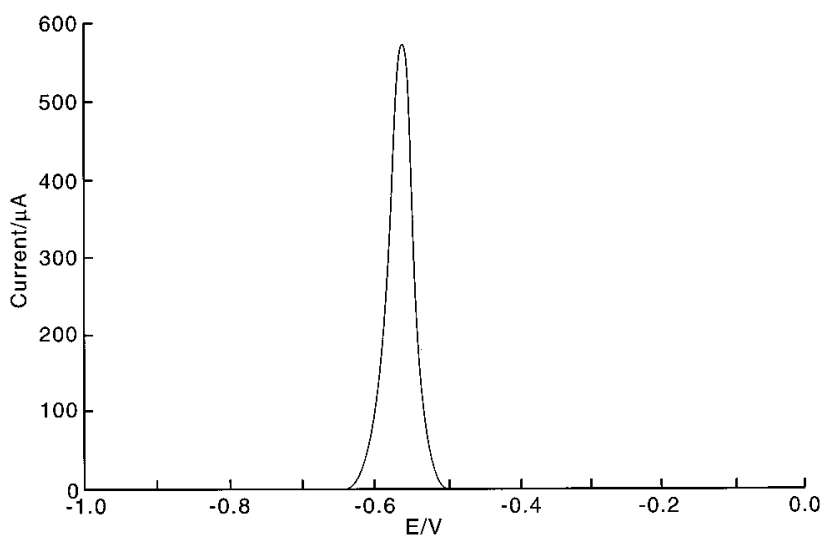

Figure 2. Simulated anodic stripping voltammogram for a wall-jet electrode possessing the parameters given in the text and for a scan rate of $100 \mathrm{mV} \mathrm{s}^{-1}$.

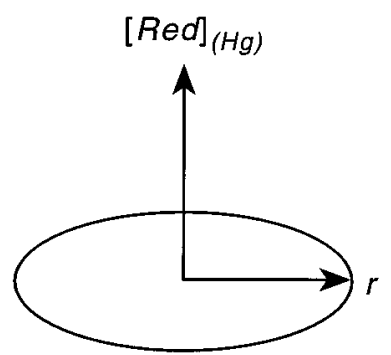

Figure 3. Coordinate system used in Figure 4.

Simulations were carried out at a potential scan rate of 100 $\mathrm{mV} \mathrm{s}^{-1}$ and the parameters given above. Under these conditions, as is evident from Figure 2, the peak is symmetric as the potential scan rate is sufficient to "outrun" the effect of convection. To visualize the concentration of Red and its radial distribution within the film, the simulation data were converted into a sequence of three-dimensional images of the form shown in Figure 3.

A cartoon of the evolution of the Red concentration is shown in Figure 4, where the circular disk represents the whole electrode and the height of a point above that surface is directly proportional to the concentration of Red. Each image corresponds to a separate potential on the scan. At potentials near -0.56 and $-0.570 \mathrm{~V}$ it is apparent that the middle of the disk is significantly depleted of Red while the electrode periphery is relatively unstripped! Thus the anticipated spatially differential stripping is a major feature of ASV at a wall-jet electrode and hitherto unappreciated. For clarity a twodimensional concentration profile of the Red distribution at $-0.55 \mathrm{~V}$ is shown in Figure 5. It is clear that the Red concentration at the center of the disk is markedly less than at the edge of the electrode.

Careful perusal of Figure 4 reveals, for potentials near -0.55 V, a "ridge" around the "hole" of concentration depleted at the disk center. The feature is confirmed by the two-dimensional plot in Figure 5, which clearly shows a buildup of material on the unstripped part of the electrode. It must be concluded that material which has been oxidized out of the mercury film becomes redeposited at greater radial distances from where it was lost. This remarkable result arises as a result of the presumed electrochemical reversibility of the redox couple and the extreme nonuniformity of the diffusion layer. Near the electrode center the latter becomes very thin and "promotes" the loss of Red by ready transport of Ox away from the surface. This material is transported away radially by the flow. On the outer parts of the disk the thick diffusion layer is much less 

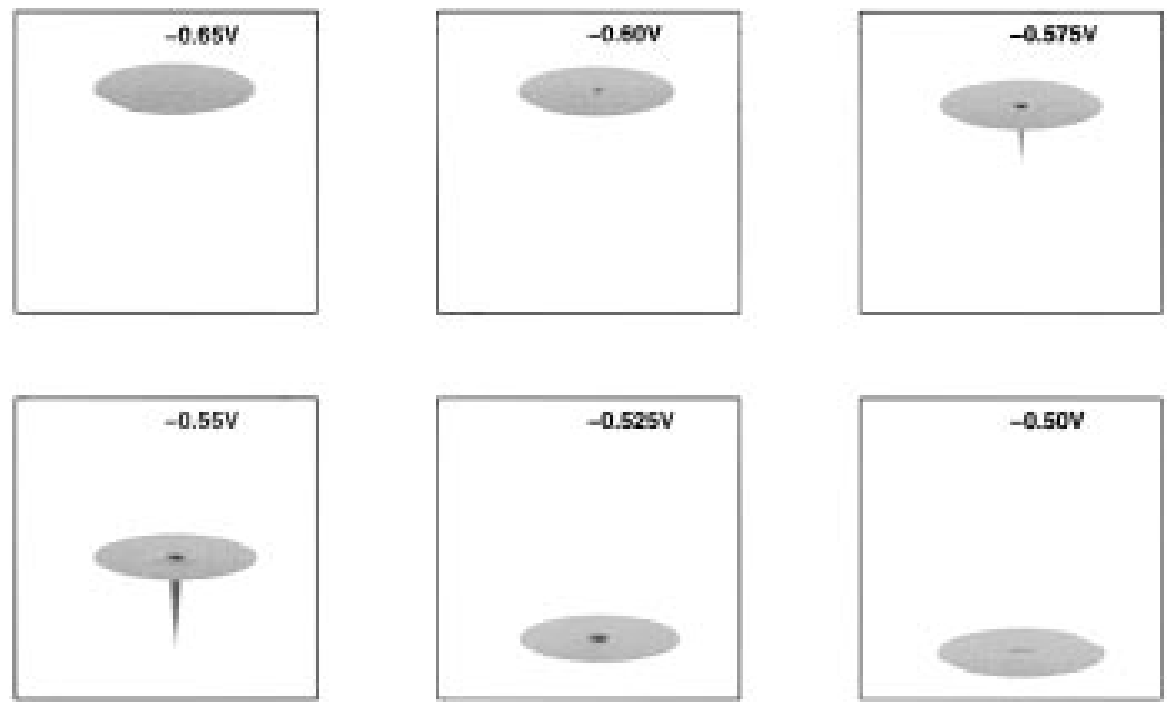

Figure 4. Red concentration profiles over the film electrode throughout a stripping peak at a wall-jet electrode for a scan rate of $100 \mathrm{mV} \mathrm{s}^{-1}$ and a flow rate of $10^{-2} \mathrm{~cm}^{3} \mathrm{~s}^{-1}$.

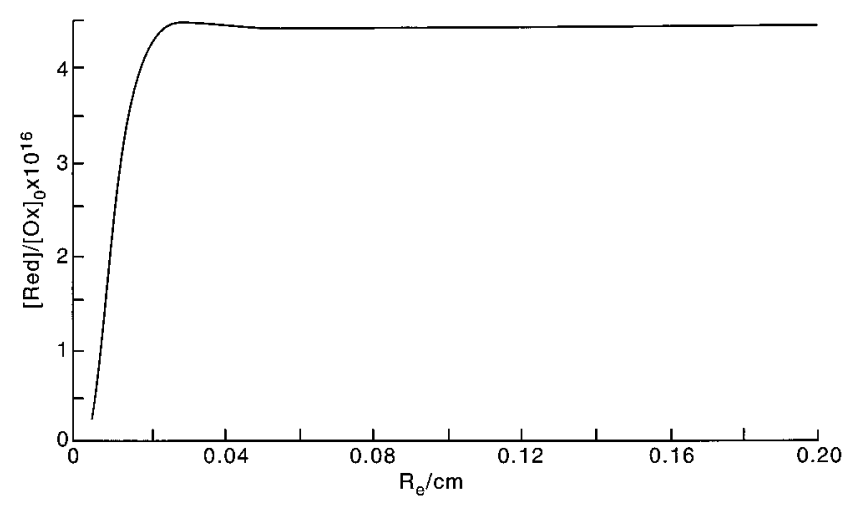

Figure 5. Concentration profile within the mercury film electrode along the radius, $r$, for the data shown in Figure 4 at a voltage of $-0.55 \mathrm{~V}$.

efficient at removing Ox so that the equilibrium

$$
\operatorname{Red}_{(\mathrm{Hg})}-n \mathrm{e}^{-} \rightleftharpoons \mathrm{Ox}_{(\text {diffusion layer) }} \stackrel{\text { mass transport }}{\longrightarrow} \mathrm{Ox}_{\text {bulk }}
$$

becomes shifted back in favor of Red.
To explore further the redeposition effect, we plot the concentration profiles of $\mathrm{Ox}$ in solution as a function of potential using $(\eta, r)$ space. Figure 6 shows this evolution; a buildup of Ox during the anodic sweep is apparent. In addition the released Ox can be seen to be progressively swept toward the edge of the electrode as a result of the convective flow. The point of maximum redeposition of Red was found to correlate with the maximum in the Ox concentration profile so that it occurred slightly "upstream" of the latter. This observation supports the explanations offered above for the presence of redeposited Red in the film.

\section{Conclusions}

The simulation of ASV at a wall-jet electrode has been successfully and quantitatively understood. The anticipated effect of electrode nonuniformity is shown first in the enhanced differential stripping of Red and second in the redeposition of this species resulting from the buildup of $\mathrm{Ox}$ in solution which is increasingly inefficiently removed by convection at larger radial distances. Note that throughout this paper we have

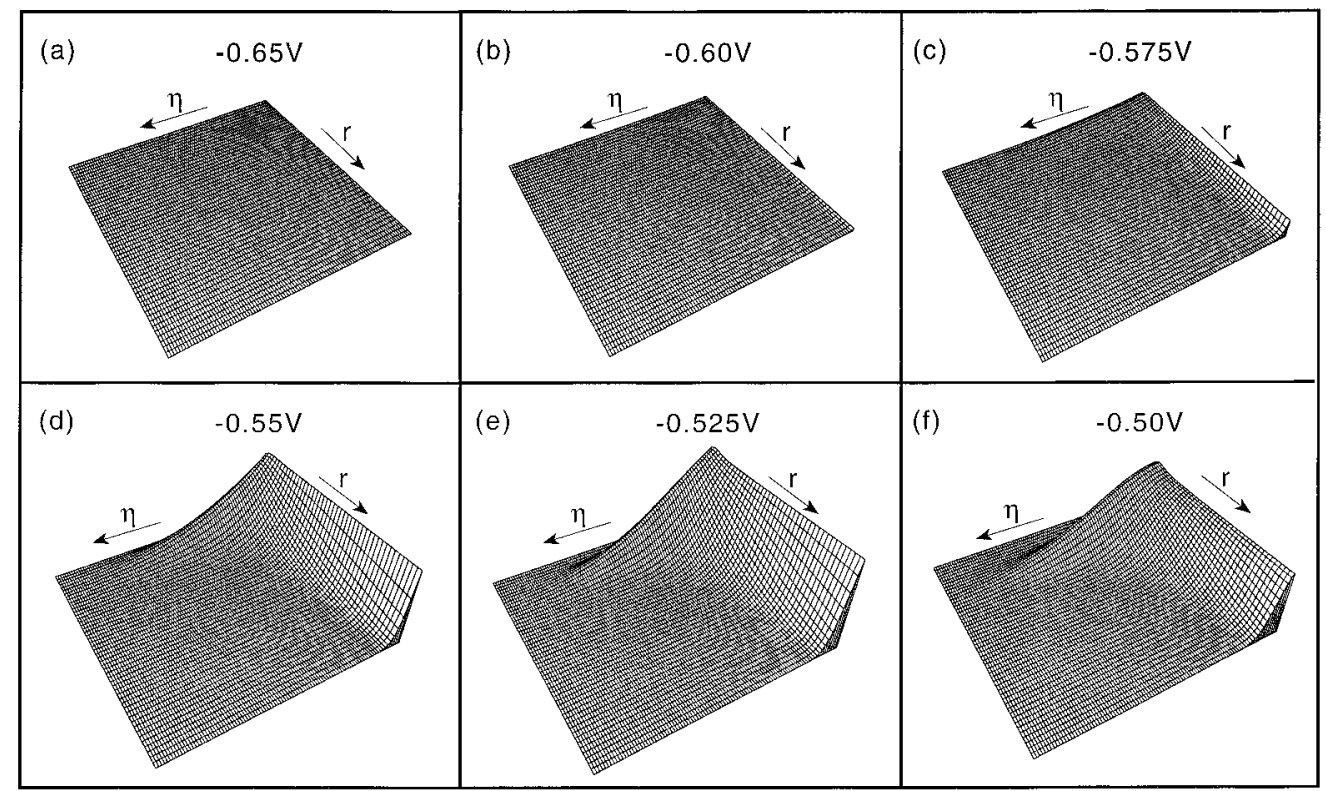

Figure 6. Three-dimensional concentration profiles of $\mathrm{Ox}$ in solution plotted in $(\eta, \mathrm{r})$ space for a scan rate of $100 \mathrm{mV} \mathrm{s}{ }^{-1}$ and a flow rate of $10^{-2}$ $\mathrm{cm}^{3} \mathrm{~s}^{-1}$. 
assumed that the Red/Ox couple is reversible; the requirement for sufficiently high electrode kinetics for this assumption to be met in hydrodynamic systems is addressed elsewhere. ${ }^{31}$

Acknowledgment. We thank the EPSRC for support under the Analytical Science Program (Grant Number GR/L/36413).

\section{References and Notes}

(1) Brainina, Kh.; Neyman, E. Electroanalytical Stripping Methods; John Wiley and Sons: New York, 1993.

(2) Wang, J. Analytical Electrochemistry; VCH: Weinheim, 1994.

(3) Batley, G. E.; Florence, T. M. J. Electroanal. Chem. 1974, 55, 23.

(4) Bakaanov, V. I.; Zakharov, M. S.; Antip'eva, V. A.; Grigorchenko, A. P. Zh. Anal. Khim., 1974, 29, 421.

(5) Brett, C. M. A.; Lima, J. L. F. C.; Quinaz Garcia, M. B. Analyst 1994, 119, 1229.

(6) Brett, C. M. A.; Neto, M. M. P. M. J. Electroanal. Chem. 1989, $258,345$.

(7) Brett, C. M. A.; Oliveira Brett, A. M.; Pereira, J. L. C. Electroanalysis 1991, 3, 83 .

(8) Neto, M. M. P. M.; Rocha, M. M. G. S.; Brett, C. M. A. Talanta 1994, 41, 1597.

(9) Brett, C. M. A.; Quinaz Garcia, M. B.; Lima, J. L. F. C. Electroanalysis 1996, 8, 1169.

(10) Lieberman, S. H.; Zirino, A. Anal. Chem. 1974, 46, 20.

(11) Andrews, R. W.; Johnson, D. C. Anal. Chem. 1974, 48, 1057.

(12) Roe, D. K.; Toni, J. E. A. Anal. Chem. 1965, 37, 1503.
(13) Brett, C. M. A.; Oliveira Brett, A. M. J. Electroanal. Chem. 1989, $262,83$.

(14) Schiewe, J.; Oldham, K. B.; Myland, J. C.; Bond, A. M.; VicenteBeckett, V. A.; Fletcher, S. Anal. Chem. 1997, 69, 2673.

(15) de Vries, W. T.; van Dalen, E. J. Electroanal. Chem. 1967, 14, 315.

(16) Vydra, F.; Stulik, K.; Juláková, E. Electrochemical Stripping Analysis; Ellis Horwood: Chichester, 1976.

(17) Ball, J. C.; Compton, R. G. Electroanalysis 1997, 9, 765.

(18) Ball, J. C.; Cooper, J. A.; Compton, R. G. J. Electroanal. Chem., in press.

(19) Glauert, M. B. J. Fluid Mech. 1956, 1, 625.

(20) Aoki, K.; Tokuda, K.; Matsuda, H. J. Electroanal. Chem. 1986, $206,37$.

(21) Albery, W. J.; Brett, C. M. A. J. Electroanal. Chem. 1983, 148, 211.

(22) Yamada, J.; Matsuda, H. J. Electroanal. Chem. 1973, 44, 189.

(23) Albery, W. J. J. Electroanal. Chem. 1985, 191, 1.

(24) Gunasingham, H.; Fleet, B. Anal. Chem. 1983, 55, 1409.

(25) Schlichting, H. Boundary Layer Theory; Pergamon Press: London, 1955; pp 73-75.

(26) Compton, R. G.; Greaves, C. R.; Waller, A. M. J. Appl. Electrochem. 1990, 20, 575.

(27) Albery, W. J.; Brett, C. M. A. J. Electroanal. Chem. 1983, 148, 201.

(28) Fisher, A. C.; Compton, R. G. J. Phys. Chem. 1991, 95, 7538.

(29) Compton, R. G.; Fisher, A. C.; Lathom, M. H.; Brett, C. M. A.; Oliveira Brett, A.M. J. Phys. Chem. 1992, 96, 8363.

(30) Weast, R. C. Handbook of Chemistry and Physics; CRC Press: Cleveland, 1994.

(31) Ball, J. C.; Compton, R. G. Electroanalysis, in press. 